\title{
Effect of Expansion-Compression Ratio on Performance of the Miller cycle
}

\author{
R. EBRAHIMI* \\ Department of Agriculture Machine Mechanics, Shahrekord University \\ P.O. Box 115, Shahrekord, Iran
}

(Received June 10, 2011)

\begin{abstract}
The objective of this study is to analyze the effect of expansion-compression ratio on the performance of dual cycle. Using finite-time thermodynamics, the relations between thermal efficiency, power output and compression ratio for an air standard Miller cycle have been derived. In the model, the nonlinear relation between the specific heats of working fluid and its temperature, the frictional loss computed according to the mean velocity of the piston, and heat transfer loss are considered. The results show that the power output first increases with the increasing expansion-compression ratio and then starts to decrease. Comparisons of the power output of the Miller, Otto, and Atkinson cycles show that if compression ratio is less than certain value, the power output for Otto cycle is higher, while if compression ratio exceeds certain value, the power output for the Miller cycle is higher. With further increase in compression ratio, the power output for Atkinson cycle is higher. In high compression ratio, the power output of the Miller cycle is higher. The results obtained in the present study provide guidance to the performance evaluation and improvement for practical internal combustion engines.
\end{abstract}

PACS: 05.70.Ln, 82.60.Fa, 88.05.-b, 88.20.td

\section{Introduction}

A series of achievements have been made since finite-time thermodynamics was used to analyze and optimize the performance of real thermodynamic processes, devices and cycles [1-5]. In 1940's, Miller [6] proposed a different Otto cycle with unequal compression and expansion stroke called the Miller cycle. The Miller cycle has been put attention recently [7], and some authors have examined the finite-time thermodynamic performance of the Miller cycle. Hatamura et al. [8] report that the Miller cycle has advantages such as higher mean effective pressure than the Otto cycle with lower nominal compression ratio. Fukuzawa et al. [9] described the main technologies and performance specifications for a high efficiency Miller cycle gas engine as well as for the series of engines planned in the future. Al-Sarkhi and Akash [10] compared the performance characteristic curves of the Atkinson cycle with those of the Miller and JouleBrayton cycles by using numerical examples, and outlined the effect of maximizing power density on the performance of the cycle efficiency. Sasaki et al. [11] report an efficient Miller cycle with a high performance capacitor system for hybrid buses. Wu et al. [12] performed a performance analysis and optimization of a supercharged

* e-mail: Rahim.Ebrahimi@gmail.com
Miller cycle Otto engine. Their zero-dimensional analysis is based on the Otto cycle. They report that the Miller cycle shows no efficiency advantage and suffers a penalty in power output in the normally aspirated version.

In the supercharged Otto engine adopted for the Miller cycle, it has again no efficiency advantage but does provide increased output with a reduced propensity to engine knock problem. Ge et al. derived the performance characteristics of the Miller cycle with heat transfer loss [13] and with heat transfer and friction-like term losses [14], respectively. These works were done without considering the variable specific heats of working fluid. Ge et al. [15] also studied the effect of variable specific heats of working fluid on performance of endoreversible Miller cycle. Chen et al. [16] built a class of generalized irreversible universal steady flow heat engine cycle model consisting of two heating branches, two cooling branches, and two adiabatic branches with consideration of the losses of heat resistance, heat leakage, and internal irreversibility. The performance characteristics of the Diesel, Otto, Brayton, Atkinson, dual and Miller cycles were derived.

As can be seen in the relevant literature, the investigations of the effect of expansion-compression ratio on performance of the Miller cycle do not appear to have been published. Therefore, the objective of this study is to examine the effect of expansion-compression ratio on the performance of air standard Miller cycle. 


\section{An irreversible Miller cycle model}

The pressure-volume $(P-V)$ and the temperature- entropy $(T-S)$ diagrams of an irreversible Miller heat engine is shown in Fig. 1 , where $T_{1}, T_{2}, T_{3}, T_{4}$, and $T_{5}$ are the temperatures of the working substance in state points $1,2,3,4$, and 5 . Process $1 \rightarrow 2$ is an isentropic compression. The heat addition occurs in the constant volume process $2 \rightarrow 3$. The process $3 \rightarrow 4$ is an isentropic expansion process. The heat addition occurs in two steps: processes $4 \rightarrow 5$ and $5 \rightarrow 1$ are constant volume and constant pressure heat additions, respectively.
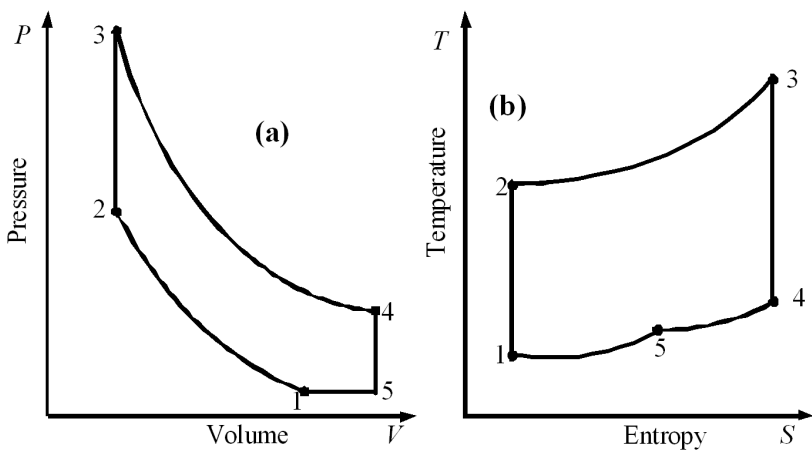

Fig. 1. (a) $P-V$ diagram; (b) $T-S$ diagram for the air standard Miller cycle.

In most cycle models, the working fluid is assumed to behave as an ideal gas with constant specific heats. But this assumption can be valid only for small temperature difference. For the large temperature difference encountered in the practical cycle, this assumption cannot be applied. According to Refs. [17, 18], for the temperature range of $300-3500 \mathrm{~K}$, the specific heat with constant volume can be written as

$$
\begin{aligned}
& C_{V}=2.506 \times 10^{-11} T^{2}+1.454 \times 10^{-7} T^{1.5} \\
& -4.245 \times 10^{-7} T+3.162 \times 10^{-5} T^{0.5}+1.0433 \\
& -1.512 \times 10^{4} T^{-1.5}+3.063 \times 10^{5} T^{-2} \\
& -2.212 \times 10^{-7} T^{-3},
\end{aligned}
$$

where $T$ is the absolute temperature and the unit of $C_{V}$ is $\mathrm{J} /(\mathrm{kg} \mathrm{K})$.

According to the relation between specific heat with constant pressure and specific heat with constant volume, the specific heat with constant volume can be written as

$$
\begin{aligned}
C_{p} & =C_{V}+R_{\mathrm{air}} \\
& =2.506 \times 10^{-11} T^{2}+1.454 \times 10^{-7} T^{1.5} \\
& -4.245 \times 10^{-7} T+3.162 \times 10^{-5} T^{0.5}+1.3303 \\
& -1.512 \times 10^{4} T^{-1.5}+3.063 \times 10^{5} T^{-2} \\
& -2.212 \times 10^{-7} T^{-3},
\end{aligned}
$$

where $R_{\text {air }}=0.287 \mathrm{~kJ} / \mathrm{kg}$ is the gas constant of the working fluid.
The heat added per second to the working fluid during process $2 \rightarrow 3$ is

$$
\begin{aligned}
& Q_{\text {in }}=\dot{m}_{t} \int_{T_{2}}^{T_{3}} C_{V} \mathrm{~d} T=\dot{m}_{t}\left(8.353 \times 10^{-12} T^{3}\right. \\
& +5.818 \times 10^{-8} T^{2.5}-2.123 \times 10^{-7} T^{2} \\
& +2.108 \times 10^{-5} T^{1.5}+1.0433 T+3.024 \times 10^{4} T^{-0.5} \\
& \left.\quad-3.063 \times 10^{5} T^{-1}+1.106 \times 10^{7} T^{-2}\right)_{T_{2}}^{T_{3}}
\end{aligned}
$$

The heat rejected per second by the working fluid during processes $4 \rightarrow 5$ and $5 \rightarrow 1$ is

$$
\begin{aligned}
& Q_{\text {out }}=\dot{m}_{t}\left[\int_{T_{5}}^{T_{4}} C_{V} \mathrm{~d} T+\int_{T_{1}}^{T_{5}} C_{p} \mathrm{~d} T\right] \\
& \quad=\dot{m}_{t}\left(8.353 \times 10^{-12} T^{3}+5.818 \times 10^{-8} T^{2.5}\right. \\
& \quad-2.123 \times 10^{-7} T^{2}+2.108 \times 10^{-5} T^{1.5} \\
& \quad+1.0433 T+3.024 \times 10^{4} T^{-0.5} \\
& \left.\quad-3.063 \times 10^{5} T^{-1}+1.106 \times 10^{7} T^{-2}\right)_{T_{5}}^{T_{4}} \\
& \quad+\dot{m}_{t}\left(8.353 \times 10^{-12} T^{3}+5.818 \times 10^{-8} T^{2.5}\right. \\
& \quad-2.123 \times 10^{-7} T^{2}+2.108 \times 10^{-5} T^{1.5} \\
& +1.3303 T+3.024 \times 10^{4} T^{-0.5} \\
& \left.-3.063 \times 10^{5} T^{-1}-1.106 \times 10^{-7} T^{-2}\right)_{T_{1}}^{T_{5}}
\end{aligned}
$$

where $\dot{m}_{t}$ is the mass flow rate of the working fluid and the unit of $\dot{m}_{t}$ is $\mathrm{kg} / \mathrm{s}$.

Since $C_{p}$ and $C_{V}$ are dependent on temperature, adiabatic exponent $\gamma=C_{p} / C_{V}$ will vary with temperature. According to Refs. [19, 20] for small change in temperature $\mathrm{d} T$, and volume $\mathrm{d} V$ of the working fluid, the equation for reversible adiabatic process with variable $\gamma$ can be written as follows:

$$
(T+\mathrm{d} T)(V+\mathrm{d} V)^{\gamma-1}=T V^{\gamma-1} .
$$

Expanding the right hand term, one arrives to an alternative form as

$$
\begin{aligned}
& (T+\mathrm{d} T)(V+\mathrm{d} V)^{\gamma-1} \\
& =(T+\mathrm{d} T)\left(V^{\gamma-1}+(\gamma-1) V^{\gamma-2} \mathrm{~d} V+\ldots \mathrm{d} Z_{0}^{2}\right. \\
& \left.+\ldots+\ldots \frac{\mathrm{d} V}{\approx 0}\right) \mathrm{d} V \\
& =(T+\mathrm{d} T)\left(V^{\gamma-1}+(\gamma-1) V^{\gamma-2} \mathrm{~d} V\right) \\
& =T V^{\gamma-1}+(\gamma-1) T V^{\gamma-2} \mathrm{~d} V+V^{\gamma-1} \mathrm{~d} T \\
& +\underbrace{(\gamma-1) V^{\gamma-2}}_{\approx 0} \mathbb{W d T} \\
& =T V^{\gamma-1}+(\gamma-1) T V^{\gamma-2} \mathrm{~d} V+V^{\gamma-1} \mathrm{~d} T .
\end{aligned}
$$

By substituting Eq. (6) into Eq. (5), we finally get

$$
C_{V} \frac{\mathrm{d} T}{T}=-R_{\text {air }} \frac{\mathrm{d} V}{V} \text {. }
$$

Integrating Eq. (7) in process $i \rightarrow j$, we have 


$$
C_{V} \ln \frac{T_{j}}{T_{i}}=R_{\text {air }} \ln \frac{V_{i}}{V_{j}},
$$

where the temperature in the equation of $C_{V}$ is defined as:

$$
T=\left(T_{j}-T_{i}\right) / \ln \left(\frac{T_{j}}{T_{i}}\right) .
$$

The expansion-compression ratio, $\psi$, the effective compression ratio, $r_{\mathrm{c}}^{*}$, and the compression ratio, $r_{\mathrm{c}}$, are defined as:

$$
\begin{aligned}
& \psi=\frac{V_{5}}{V_{1}}=\frac{T_{5}}{T_{1}}, \\
& r_{\mathrm{c}}^{*}=\frac{V_{1}}{V_{2}}
\end{aligned}
$$

and

$$
r_{\mathrm{c}}=\frac{V_{5}}{V_{2}}=\psi r_{\mathrm{c}}^{*} .
$$

Therefore, equations for reversible adiabatic processes $1 \rightarrow 2$ and $3 \rightarrow 4$ are as follows:

$$
\begin{aligned}
& C_{V} \ln \frac{T_{2}}{T_{1}}=R_{\text {air }} \ln r_{\mathrm{c}}^{*}, \\
& C_{V} \ln \frac{T_{3}}{T_{4}}=R_{\text {air }} \ln \left(\psi r_{\mathrm{c}}^{*}\right) .
\end{aligned}
$$

For an ideal reciprocating heat engine cycle model, there are no losses. However, for a real reciprocating heat engine cycle, heat transfer irreversibility between the working fluid and the cylinder wall is not negligible. It can be assumed that the heat loss through the cylinder wall is proportional to the average temperature of both the working fluid and the cylinder wall and that the wall temperature is constant at $T_{0}[21-23]$. If the released heat by combustion for one mass working fluid is $A$ and the heat leakage coefficient of the cylinder wall is $B$, the heat added to the working fluid by combustion is given by the linear relation

$$
\dot{Q}_{\text {in }}=\dot{m}_{t}\left[A-B\left(T_{2}+T_{4}-2 T_{0}\right)\right] .
$$

Every time the piston moves, friction acts to retard the motion. Considering the friction effects on the piston in all the processes of the cycle, we assume a dissipation term represented by a friction force that is linearly proportional to the velocity of the piston, which can be written as follows [24-26]:

$$
f_{\mu}=\mu \nu=\mu \frac{\mathrm{d} x}{\mathrm{~d} t},
$$

where $\mu$ is a coefficient of friction that takes into account the global losses and $x$ is the piston displacement. Therefore, the lost power is

$$
P_{\mu}=\frac{\mathrm{d} W_{\mu}}{\mathrm{d} t}=\mu \frac{\mathrm{d} x}{\mathrm{~d} t} \frac{\mathrm{d} x}{\mathrm{~d} t}=\mu \nu^{2} .
$$

The mean velocity of the piston is

$$
\bar{\nu}=4 L N=4 L^{*}\left[1+\frac{r_{\mathrm{c}}^{*}(\psi-1)}{r_{\mathrm{c}}^{*}-1}\right] N,
$$

where $L$ is total distance of the piston traveling per cycle and $L^{*}$ is the length of stroke compression.
The power output of the cycle is

$$
\begin{aligned}
& P_{\mathrm{du}}=\dot{Q}_{\text {in }}-\dot{Q}_{\mathrm{out}}-P_{\mu} \\
& \quad=\dot{m}_{t}\left[8.353 \times 10^{-12}\left(T_{1}^{3}+T_{3}^{3}-T_{2}^{3}-T_{4}^{3}\right)\right. \\
& \quad+5.818 \times 10^{-8}\left(T_{1}^{2.5}+T_{3}^{2.5}-T_{2}^{2.5}-T_{4}^{2.5}\right) \\
& \quad-2.123 \times 10^{-7}\left(T_{1}^{2}+T_{3}^{2}-T_{2}^{2}-T_{4}^{2}\right) \\
& \quad+2.108 \times 10^{-5}\left(T_{1}^{1.5}+T_{3}^{1.5}-T_{2}^{1.5}-T_{4}^{1.5}\right) \\
& +1.0433\left(T_{3}-T_{2}+T_{5}-T_{4}\right)+1.3303\left(T_{1}-T_{5}\right) \\
& +3.024 \times 10^{4}\left(T_{1}^{-0.5}+T_{3}^{-0.5}-T_{2}^{-0.5}-T_{4}^{-0.5}\right) \\
& \quad-3.063 \times 10^{5}\left(T_{1}^{-1}+T_{3}^{-1}-T_{2}^{-1}-T_{4}^{-1}\right) \\
& \left.\quad+1.106 \times 10^{7}\left(T_{1}^{-2}+T_{3}^{-2}-T_{2}^{-2}-T_{4}^{-2}\right)\right] \\
& \quad-16 \mu\left(L^{*}\left[1+\frac{r_{\mathrm{c}}^{*}(\psi-1)}{r_{\mathrm{c}}^{*}-1}\right] N\right)^{2} .
\end{aligned}
$$

The efficiency of the cycle is

$$
\begin{aligned}
& \eta_{\mathrm{du}}=\frac{P_{\mathrm{du}}}{\dot{Q}_{\mathrm{in}}} \\
& =\left\{\dot { m } _ { t } \left[8.353 \times 10^{-12}\left(T_{1}^{3}+T_{3}^{3}-T_{2}^{3}-T_{4}^{3}\right)\right.\right. \\
& +5.818 \times 10^{-8}\left(T_{1}^{2.5}+T_{3}^{2.5}-T_{2}^{2.5}-T_{4}^{2.5}\right) \\
& -2.123 \times 10^{-7}\left(T_{1}^{2}+T_{3}^{2}-T_{2}^{2}-T_{4}^{2}\right) \\
& +2.108 \times 10^{-5}\left(T_{1}^{1.5}+T_{3}^{1.5}-T_{2}^{1.5}-T_{4}^{1.5}\right) \\
& +1.0433\left(T_{3}-T_{2}+T_{5}-T_{4}\right)+1.3303\left(T_{1}-T_{5}\right) \\
& +3.024 \times 10^{4}\left(T_{1}^{-0.5}+T_{3}^{-0.5}-T_{2}^{-0.5}-T_{4}^{-0.5}\right) \\
& -3.063 \times 10^{5}\left(T_{1}^{-1}+T_{3}^{-1}-T_{2}^{-1}-T_{4}^{-1}\right) \\
& \left.+1.106 \times 10^{7}\left(T_{1}^{-2}+T_{3}^{-2}-T_{2}^{-2}-T_{4}^{-2}\right)\right] \\
& \left.-16 \mu\left(L^{*}\left[1+\frac{r_{\mathrm{c}}^{*}(\psi-1)}{r_{\mathrm{c}}^{*}-1}\right] N\right)^{2}\right\} \\
& \int\left\{\dot { m } _ { t } \left[8.353 \times 10^{-12}\left(T_{3}^{3}-T_{2}^{3}\right)\right.\right. \\
& +5.818 \times 10^{-8}\left(T_{3}^{2.5}-T_{2}^{2.5}\right) \\
& -2.123 \times 10^{-7}\left(T_{3}^{2}-T_{2}^{2}\right) \\
& +2.108 \times 10^{-5}\left(T_{3}^{1.5}-T_{2}^{1.5}\right)+1.0433\left(T_{3}-T_{2}\right) \\
& +3.024 \times 10^{4}\left(T_{3}^{-0.5}-T_{2}^{-0.5}\right) \\
& -3.063 \times 10^{5}\left(T_{3}^{-1}-T_{2}^{-1}\right) \\
& \left.\left.+1.106 \times 10^{7}\left(T_{3}^{-2}-T_{2}^{-2}\right)\right]\right\} .
\end{aligned}
$$

When $\psi=1$ or $T_{5}=T_{1}$, the Miller cycle becomes Otto cycle, while $T_{5}=T_{4}$, the Miller cycle becomes the Atkinson cycle.

When $r_{\mathrm{c}}^{*}, \psi$ and $T_{1}$ are given, $T_{2}$ can be obtained from Eq. (13); then, substituting from Eq. (3) into Eq. (15) yields $T_{3}$; and $T_{4}$ can be found from Eq. (14); at last, $T_{5}$ can be found by Eq. (10). Substituting $T_{1}, T_{2}, T_{3}, T_{4}$, and $T_{5}$ into Eqs. (19) and (20) yields the power output and thermal efficiency. Therefore, the relations between 
the power output, the thermal efficiency and the compression ratio can be derived.

\section{Results and discussion}

The following constants and parameter values have been used in this exercise: $L^{*}=72 \mathrm{~mm}, T_{1}=300 \mathrm{~K}$, $\mu=12.9 \mathrm{~N} \mathrm{~s} m^{-1}, B=1.4 \mathrm{~kJ} /(\mathrm{kg} \mathrm{K}), N=4200 \mathrm{rpm}$, $A=2820.5 \mathrm{~kJ} / \mathrm{kg}, \psi=1-1.7$, and $M=0.016 \mathrm{~kg} / \mathrm{s}$ [27-30]. Using the above constants and range of parameters, the power output versus compression ratio characteristic, the thermal efficiency versus compression ratio characteristic and the power output versus thermal efficiency characteristic with varying the expansion-compression ratio can be plotted as in Figs. 2-4 (the dashed lines in the figures denote where the cycle cannot work because $T_{5}$ exceeds $T_{4}$ ). One can see that the power output versus compression ratio characteristic and the efficiency versus compression ratio characteristic are parabolic like curves, and the power output versus efficiency curve is loop shaped. They reflect the performance characteristics of a real irreversible Miller cycle. Numerical examples are shown as follows.

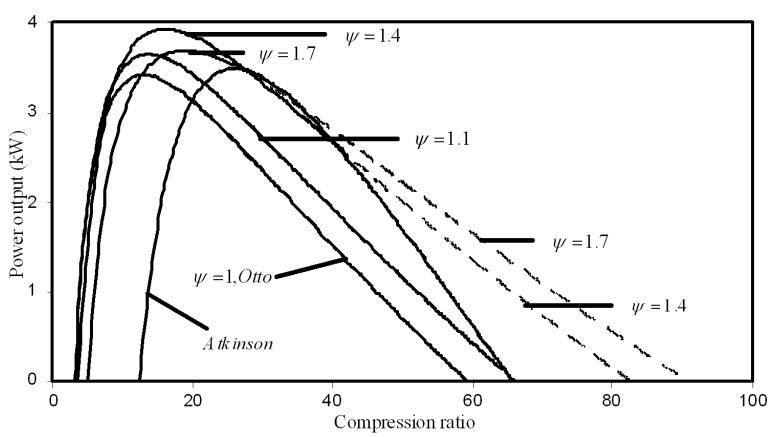

Fig. 2. Effect of expansion-compression ratio on the variation of the power output with compression ratio.

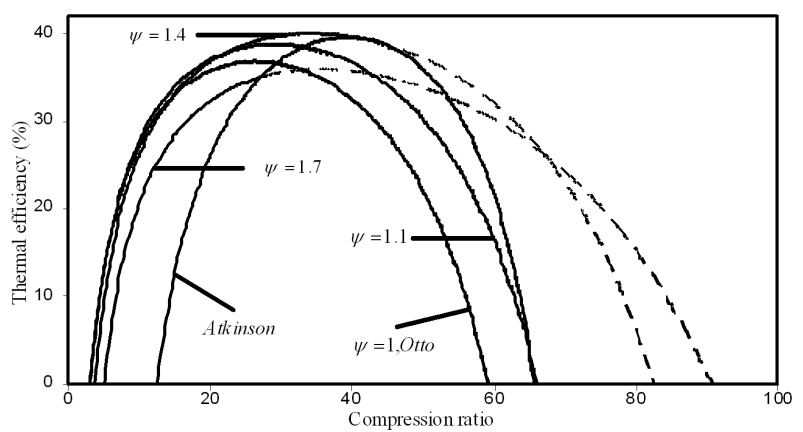

Fig. 3. Effect of expansion-compression ratio on the variation of the power output with compression ratio.

Figures 2-4 show the effect of expansion-compression ratio versus compression ratio variation on the performance of cycle by considering the nonlinear relation be-

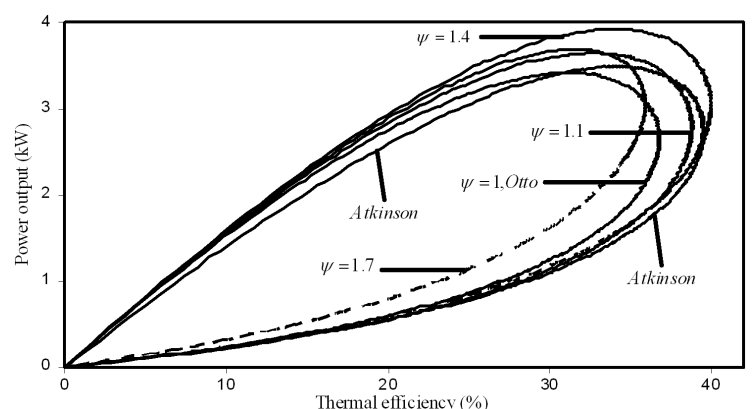

Fig. 4. Effect of expansion-compression ratio on the variation of the power output with thermal efficiency.

tween the specific heats of working fluid and its temperature, the frictional loss and heat transfer loss. From these figures, it can be found that the expansion-compression ratio plays important roles on the performance cycle. It is clearly seen that the effect of expansion-compression ratio on the power output and thermal efficiency is related to compression ratio. The results shows that if compression ratio is less than certain value, the power output decreases with increasing expansion-compression ratio, while if compression ratio exceeds certain value, the power output first increases and then starts to decrease with increasing expansion-compression ratio. With further increase in compression ratio, the increase of expansion-compression ratio results in increasing the power output. The behavior of the thermal efficiency versus compression ratio for various expansion-compression ratio is qualitatively similar to that for the power output.

From these figures, we can conclude that when expansion-compression ratio increases from 1.1 to 1.4 , the maximum power output and the maximum thermal efficiency increase by $7.7 \%$ and $3.1 \%$, respectively. With further increase in expansion-compression ratio, from 1.4 to 1.7, the maximum power output and the maximum thermal efficiency decrease by $6.5 \%$ and 11.4 , respectively. The optimal compression ratio corresponding to maximum power output point, the optimal compression ratio corresponding to maximum thermal efficiency point and the working range of the cycle increase with increasing expansion-compression ratio. The results also show that the optimal thermal efficiency corresponding to maximum power output and the optimal power output corresponding to maximum thermal efficiency first increase and then start to decline with increasing expansion-compression ratio. Numerical calculation shows that for any same compression ratio, the largest power output is for Otto when $r_{\mathrm{c}} \leq 4.3$, is for Miller when $4.3<r_{\mathrm{c}}<27.5$ and $r_{\mathrm{c}}>40.5$ and is for Atkinson when $27.5 \leq r_{\mathrm{c}} \leq 40.5$ and also the largest thermal efficiency is for Otto when $r_{\mathrm{c}} \leq 5.3$ and is for Miller when $5.3<r_{\mathrm{c}} \leq 40.7$ and $r_{\mathrm{c}}>44.5$ and is for Atkinson when $40.7<r_{\mathrm{c}} \leq 44.5$.

According to above analysis, it can be found that the effect of the expansion-compression ratio on the cycle performance is obvious, and it should be considered in 
practice cycle analysis in order to make the cycle model be more close to practice.

\section{Conclusions}

In this study, finite-time thermodynamics analysis of an irreversible air standard Miller cycle is established which is closer to practice engine. In this model, effect of expansion-compression ratio by considering the nonlinear relation between the specific heats of working fluid and its temperature, the frictional loss and heat transfer loss is analyzed. Numerical examples displayed the influences of this parameter in power output and efficiency of the cycle. The general conclusions drawn from the results of this work are as follows:

- If compression ratio is less than certain value, the power output decreases with increasing expansion-compression ratio, while if compression ratio exceeds certain value, the power output first increases and then starts to decrease with increasing expansion-compression ratio. With further increase in compression ratio, the increase of expansion-compression ratio results in increasing the power output. The behavior of the thermal efficiency versus compression ratio for various expansion-compression ratio is qualitatively similar to that for the power output.

- The maximum power output, the maximum thermal efficiency, the optimal thermal efficiency corresponding to maximum power output and the optimal power output corresponding to maximum thermal efficiency first increase and then start to decline with the increasing expansion-compression ratio.

- Comparisons of the power output of the Miller, Otto, and Atkinson cycles show that if compression ratio is less than certain value, the power output for Otto cycle is higher, while if compression ratio exceeds certain value, the power output for the Miller cycle is higher. With further increase in compression ratio, the power output for the Atkinson cycle is higher. In high compression ratio, the power output of the Miller cycle is higher. The behavior of the thermal efficiency versus compression ratio for the Miller, Otto and Atkinson cycles is qualitatively similar to that for the power output.

The conclusions of this investigation are of importance when considering the designs of actual Miller engines.

\section{References}

[1] B. Andresen, P. Salamon, R.S. Berry, Phys. Today 38, 62 (1984).
[2] L. Chen, C. Wu, F. Sun, J. Non-Equilib. Thermodyn. 24, 327 (1999)

[3] L. Chen, F. Sun, Advances in Finite Time Thermodynamics: Analysis and Optimization, Nova Sci. Publ., New York 2004.

[4] R. Ebrahimi, J. Energy Instit. 84, 30 (2011).

[5] R. Ebrahimi, J. Energy Inst. 84, 38 (2011).

[6] R.H. Miller, ASME Trans. 69, 453 (1947).

[7] U. Kesgin, Int. Energy Res. 29, 189 (2005).

[8] K. Hatamura, M. Hayakawa, T. Goto, M. Hitomi, JSAE Rev. 18, 101 (1997).

[9] Y. Fukuzawa, H. Shimoda, Y. Kakuhama, H. Endo, K. Tanaka, Techn. Rev. 38, 146 (2001).

[10] A. Al-Sarkhi, B.A. Akash, Int. Commun. Heat Mass Transfer 29, 1159 (2002)

[11] M. Sasaki, S. Araki, T. Miyata, T. Kawaji, JSAE Rev. 23, 451 (2002).

[12] C. Wu, P.V. Puzinauskas, J.S. Tsai, Appl. Therm. Eng. 23, 511 (2003).

[13] Y. Ge, L. Chen, F. Sun, C. Wu, Int. Comm. Heat Mass Transfer 32, 1045 (2005).

[14] Y. Ge, L. Chen, F. Sun, C. Wu, Appl. Energy 81, 397 (2005).

[15] Y. Ge, L. Chen, F. Sun, C. Wu, Int. J. Amb. Energy 26, 203 (2005).

[16] L. Chen, W. Zhang, F. Sun, Appl. Energy 84, 512 (2007).

[17] Y. Ge, L. Chen, F. Sun, Appl. Energy 85, 618 (2008).

[18] R. Sonntag, C. Borgnakke, G. Van Wylen, Fundamentals of Thermodynamics, 5th ed., Wiley, New York 1998.

[19] Y.-L. Ge, L. Chen, F.-R. Sun, Proc. IMechE, Part D: J. Automob. Eng. 222, 887 (2008).

[20] Y. Ge, L. Chen, F. Sun, Appl. Energy 85, 618 (2008).

[21] R. Ebrahimi, J. Am. Sci. 5, 58 (2009).

[22] L. Chen, F. Zeng, F. Sun, C. Wu, Energy 21, 1201 (1996).

[23] S.A. Klein, Trans. ASME J. Eng. Gas Turbines Power 113, 511 (1991)

[24] R. Ebrahimi, J. Energy Instit. 83, 1 (2010).

[25] A. Al-Sarkhi, J.O. Jaber, M. Abu-Qudais, S.D. Probert, Appl. Energy 83, 153 (2006).

[26] L. Chen, T. Zheng, F. Sun, C. Wu, Int. J. Amb. Energy 24, 195 (2003).

[27] R. Ebrahimi, Acta Phys. Pol. A 117, 887 (2010).

[28] R. Ebrahimi, Acta Phys. Pol. A 118, 534 (2010).

[29] Y. Ge, L. Chen, F. Sun, C. Wu, J. Energy Instit. 80 , 52 (2007).

[30] Y. Ust, B. Sahin, A. Kodal, J. Energy Instit. 82, 48 (2009). 